\title{
Numerical Solution of Fractional Model of HIV-1 Infection in Framework of Different Fractional Derivatives
}

\author{
Sara Salem Alzaid, ${ }^{1}$ Badr Saad T. Alkahtani, ${ }^{2}$ Shivani Sharma, ${ }^{3}$ and Ravi Shanker Dubey $\mathbb{D}^{3}$ \\ ${ }^{1}$ Department of Mathematics, King Saud University, P.O. Box 2455, Riyadh 11451, Saudi Arabia \\ ${ }^{2}$ Mathematics Department, College of Science, King Saud University, P.O. Box 1142, Riyadh 11989, Saudi Arabia \\ ${ }^{3}$ Department of Mathematics, AMITY School of Applied Science, AMITY University Rajasthan, Jaipur 302002, India
}

Correspondence should be addressed to Ravi Shanker Dubey; ravimath13@gmail.com

Received 28 December 2020; Revised 22 February 2021; Accepted 5 March 2021; Published 22 March 2021

Academic Editor: Mohamed Boussairi Jleli

Copyright (c) 2021 Sara Salem Alzaid et al. This is an open access article distributed under the Creative Commons Attribution License, which permits unrestricted use, distribution, and reproduction in any medium, provided the original work is properly cited.

\begin{abstract}
In this paper, we have extended the model of HIV-1 infection to the fractional mathematical model using Caputo-Fabrizio and Atangana-Baleanu fractional derivative operators. A detailed proof for the existence and the uniqueness of the solution of fractional mathematical model of HIV-1 infection in Atangana-Baleanu sense is presented. Numerical approach is used to find and study the behavior of the solution of the stated model using different derivative operators, and the graphical comparison between the solutions obtained for the Caputo-Fabrizio and the Atangana-Baleanu operator is presented to see which fractional derivative operator is more efficient.
\end{abstract}

\section{Introduction}

HIV stands for the Human Immunodeficiency Virus. This virus attacks a person's immune system. The immune system of a person's body works as a defence mechanism against infections such as bacteria and viruses.

All viruses proliferate by infecting the living cells of the body. HIV targets such immune system cells which are meant to defend the body. These cells are known as $\mathrm{CD} 4^{+}$ $\mathrm{T}$ cells. $\mathrm{CD} 4^{+} \mathrm{T}$ cells are basically white blood cells that fight infection. $\mathrm{CD}^{+} \mathrm{T}$ cell count is a measure of immune function in a patient with HIV. It is an imperative determinant for the need for opportunistic infection (OI) prophylaxis. $\mathrm{CD}^{+} \mathrm{T}$ cell count is determined from blood as a part of laboratory monitoring for HIV infection.

When HIV takes over these cells, it transforms a cell into a virus factory. It makes the cell to produce thousands of copies of the virus, and these copies then infect the other $\mathrm{CD}^{+} \mathrm{T}$ cells. The infected cells do not function desirably and die early. The paucity of $\mathrm{CD}^{+} \mathrm{T}$ cells deteriorates the immune system and makes it challenging for the body to stay healthy.
$\mathrm{CD} 4^{+} \mathrm{T}$ cell count is generally measured after being diagnosed with HIV (at baseline). It is measured in every three to six months during the first two years until these cell counts in a patient's body increase above 300 cells $/ \mathrm{mm}^{3}$; else, it is measured in every twelve months. Most HIV patients can expect an average increase of about $50-100$ cells $/ \mathrm{mm}^{3}$. Patients who indulge in therapy with low $\mathrm{CD} 4{ }^{+} \mathrm{T}$ cell count or at an older age may not experience the same increase in their $\mathrm{CD} 4^{+} \mathrm{T}$ cell count despite virologic suppression.

There are a lot of factors that affect these cell counts. Medication is one of the options to keep these T cell counts high.

The characteristics of Acquired Immunodeficiency Syndrome (AIDS) pathogenesis are progressive depletion of $\mathrm{CD} 4^{+} \mathrm{T}$ cell population in close association with progressive impairment of cellular immunity and increased susceptibility to opportunistic infections (OI). HIV was originally hypothesized to be a consequence of sluggish $\mathrm{CD}^{+} \mathrm{T}$ cell destruction. However, massive $\mathrm{CD}^{+}$memory $\mathrm{T}$ cell destruction now occurs at a primitive level of infection. In most individuals, the initial destruction is countered by $\mathrm{CD} 4^{+}$memory $\mathrm{T}$ cell regeneration that protects these $\mathrm{T}$ cell numbers and 
functions above the threshold associated with overt immunodeficiency (see $[1,2])$.

The mathematical model of HIV-1 infection of $\mathrm{CD}^{+}{ }^{+} \mathrm{T}$ cells is given as follows

$$
\begin{aligned}
& \frac{d U}{d t}=\varepsilon-d U-\alpha U V, U(0)=\gamma_{1}, \\
& \frac{d I}{d t}=\alpha U V-s I, I(0)=\gamma_{2}, \\
& \frac{d V}{d t}=c I-\delta V, V(0)=\gamma_{3},
\end{aligned}
$$

here, $U(t)$ represents the population with uninfected $\mathrm{CD} 4^{+} \mathrm{T}$ cells, $I(t)$ represents the population with infected $\mathrm{CD} 4^{+} \mathrm{T}$ cells, and $V(t)$ represents the density of virions in plasma at time " $t$ ", respectively. Further, $\varepsilon$ is the rate of generation of $\mathrm{CD} 4^{+} \mathrm{T}$ cells, $d$ is the natural death rate, $\alpha$ is the infected death rate of $C D 4^{+}$T cells, $s$ is the rate by which viruses make the cells dead, $c$ is the rate of formation of virions viruses due to infected $\mathrm{CD} 4^{+} \mathrm{T}$ cells, and $\delta$ gives the death rate of viruses (see [3]). In the current work, we will be concerned with the fractional mathematical model of HIV-1 infection. Most biological systems have aftereffects or memory, so the modeling of such biological systems using fractional order derivatives have many advantages in which the effects like memory are neglected. It has been concluded that there is fractional order electrical conductance in the cell membrane of many biological organisms, and they are classified in groups of noninteger models. Thus, fractional derivatives, see also ([4-12]) study the behavior of these biological models more efficiently. Fractional calculus have been successfully growing and giving fruitful and countless developments in fields of chemistry, physics, biochemistry, medicine, biology, etc. (see [13-16]). In this work, we will consider the fractional mathematical model of HIV-1 infection in sense of Caputo-Fabrizio derivative operator [17] and Atangana-Baleanu derivative operator [18]. A detailed proof for the existence and the uniqueness of the solution is presented. The numerical solutions are presented for the fractional mathematical model in sense of the Caputo-Fabrizio and the Atangana-Baleanu derivative operator. Further, the results obtained are compared graphically to see which fractional derivative operator gives the better result.

Definition 1 (see [17]). Let $f$ be an integrable function on $\mathbb{R}$, $t>0,0<\beta<1$, the Caputo-Fabrizio fractional derivative of order $\beta$ is defined as

$$
{ }_{0}^{C F} \zeta_{t}^{\beta}(f(t))=\frac{N(\beta)}{1-\beta} \int_{0}^{t} \exp \left(\frac{-\beta(t-\tau)}{1-\beta}\right) f^{\prime}(\tau) d \tau
$$

where ${ }_{0}^{C F} \zeta_{t}^{\beta}$ represents the Caputo-Fabrizio fractional derivative of order $\beta$ and $N(\beta)$ is a normalization function and the following holds $N(0)=N(1)=1$.
Definition 2 (see [19]). Let $f$ be an integrable function on $\mathbb{R}$, $t>0,0<\beta<1$, the Caputo-Fabrizio time fractional integral of order $\beta$ is given as

$$
\mathscr{I}_{t}^{\mathscr{C} \mathscr{F} \beta}(f(t))=\frac{2(1-\beta)}{(2-\beta) N(\beta)} f(t)+\frac{2 \beta}{(2-\beta) N(\beta)} \int_{0}^{t} f(\tau) d \tau,
$$

where $N(\beta)$ is the normalization function and the following holds $N(0)=N(1)=1$.

Definition 3 (see [18]). Let $f$ be an integrable function on $\mathbb{R}$, let $0<\beta<1$, then the Atangana-Baleanu fractional derivative is given as

$$
{ }_{0}^{A B C} \zeta_{t}^{\beta}(f(t))=\frac{N(\beta)}{1-\beta} \int_{0}^{t} f^{\prime}(\tau) E_{\beta}\left[-\beta \frac{(t-\tau)^{\beta}}{1-\beta}\right] d \tau,
$$

where ${ }_{0}^{A B C} \zeta_{t}^{\beta}$ is the Atangana-Baleanu fractional derivative of order $\beta$ in Caputo sense, $E_{\beta}$ is the Mittag-Leffler function, and $N(\beta)$ is the normalization function such that $N(0)=N$ $(1)=1$.

Definition 4 (see [18]). Let $f$ be an integrable function on $\mathbb{R}$, the fractional integral of Atangana-Baleanu fractional derivative of order $\beta$ is given as

$$
\mathscr{J}_{t}^{\mathscr{A} \mathscr{B}}{ }_{t}^{\beta}(f(t))=\frac{1-\beta}{N(\beta)} f(t)+\frac{\beta}{N(\beta) \Gamma(\beta)} \int_{0}^{t} f(\tau)(t-\tau)^{\beta-1} d \tau .
$$

Theorem 5 (see [18]). The fractional differential equation

$$
\mathscr{I}_{t}^{\mathscr{A} \mathscr{B}}{ }_{t}^{\beta}(f(t))=v(t)
$$

possesses a solution which is unique given as

$$
f(t)=\frac{1-\beta}{M(\beta)} v(t)+\frac{\beta}{M(\beta) \Gamma(\beta)} \int_{0}^{t} v(\tau)(t-\tau)^{\beta-1} d \tau .
$$

\section{Fractional HIV-1 Model in Caputo- Fabrizio Sense}

The fractional mathematical model of HIV-1 infection in Caputo-Fabrizio sense is given as follows.

$$
\begin{gathered}
{ }_{0}^{C F} \zeta_{t}^{\beta} U(t)=\varepsilon-d U-\alpha U V, U(0)=\gamma_{1}, \\
\quad{ }_{0}^{C F} \zeta_{t}^{\beta} I(t)=\alpha U V-s I, I(0)=\gamma_{2}, \\
\quad{ }_{0}^{C F} \zeta_{t}^{\beta} V(t)=c I-\delta V, V(0)=\gamma_{3},
\end{gathered}
$$

here, ${ }_{0}^{\mathrm{CF}} \zeta_{t}^{\beta}$ denotes the Caputo-Fabrizio fractional derivative of order $\beta$. 
2.1. Derivation of Numerical Scheme in Sense of CaputoFabrizio Derivative. To illustrate the method ([20, 21]), we contemplate the following general equation:

$$
{ }_{0}^{C F} \zeta_{t}^{\beta} \eta(t)=g(t, \eta(t)), t \geq 0, \eta(0)=\eta_{0}
$$

where $\eta(t)$ stands for $U(t), I(t), V(t)$ and $g(t, \eta(t))$ is an integrable function that stands for $\varepsilon-d U-\alpha U V, \alpha U V-s I$ , and $c I-\delta V$, respectively. Using the fundamental theorem, the above equation can be written as

$$
\eta(t)-\eta(0)=\frac{1-\beta}{N(\beta)} g(t, \eta(t))+\frac{\beta}{N(\beta)} \int_{0}^{t} g(\tau, \eta(\tau)) d \tau .
$$

At point $t=t_{k+1}$, for $k=0,1,2 \cdots$, equation (10) becomes $\eta\left(t_{k+1}\right)-\eta(0)=\frac{1-\beta}{N(\beta)} g\left(t_{k}, \eta\left(t_{k}\right)\right)+\frac{\beta}{N(\beta)} \int_{0}^{t_{k+1}} g(\tau, \eta(\tau)) d \tau$.

At point $t=t_{k}$, for $k=0,1,2 \cdots$, equation (10) becomes $\eta\left(t_{k}\right)-\eta(0)=\frac{1-\beta}{N(\beta)} g\left(t_{k-1}, \eta\left(t_{k-1}\right)\right)+\frac{\beta}{N(\beta)} \int_{0}^{t_{k}} g(\tau, \eta(\tau)) d \tau$.

From the above two equations, we get

$$
\begin{aligned}
\eta\left(t_{k+1}\right)-\eta\left(t_{k}\right)= & \frac{1-\beta}{N(\beta)}\left[g\left(t_{k}, \eta\left(t_{k}\right)\right)-g\left(t_{k-1}, \eta\left(t_{k-1}\right)\right)\right] \\
& +\frac{\beta}{N(\beta)} \int_{t_{k}}^{t_{k+1}} g(\tau, \eta(\tau)) d \tau .
\end{aligned}
$$

Considering $\mathrm{g}(\tau, \eta(\tau))$ through Lagrange polynomial interpolation,

$$
q_{n}=g(\tau, \eta(\tau))=\frac{\tau-t_{i-1}}{t_{i}-t_{i-1}} g\left(t_{i}, \eta_{t_{i}}\right)+\frac{\tau-t_{i}}{t_{i-1}-t_{i}} g\left(t_{i-1}, \eta_{t_{i-1}}\right),
$$

where $\eta\left(t_{i}\right)$ is a function at time $t_{i}$ and $\eta\left(t_{i-1}\right)$ is a function at time $t_{i-1}$. Substituting the value of $g(\tau, \eta(\tau))$ in equation (13), we get

$$
\begin{aligned}
\eta_{k+1}-\eta_{k}= & \frac{1-\beta}{N(\beta)}\left[g\left(t_{k}, \eta\left(t_{k}\right)\right)-g\left(t_{k-1}, \eta\left(t_{k-1}\right)\right)\right] \\
& +\frac{\beta}{N(\beta)} \int_{t_{k}}^{t_{k+1}}\left(\frac{g\left(t_{k}, \eta_{k}\right)}{h}\left(\tau-t_{k-1}\right)-\frac{g\left(t_{k-1}, \eta_{k-1}\right)}{h}\left(\tau-t_{k}\right)\right) d \tau .
\end{aligned}
$$

Substituting $h=t_{i}-t_{i-1}$ and on solving, we get

$$
\begin{aligned}
\eta_{k+1}= & \eta_{0}+\left(\frac{1-\beta}{N(\beta)}+\frac{3 h}{2 N(\beta)}\right) g\left(t_{k}, \eta\left(t_{k}\right)\right) \\
& -\left(\frac{1-\beta}{N(\beta)}+\frac{\beta h}{2 N(\beta)}\right) g\left(t_{k-1}, \eta\left(t_{k-1}\right)\right) .
\end{aligned}
$$

Using the aforementioned concept of numerical scheme, the numerical scheme for the fractional model of HIV-1 infection in framework of Caputo-Fabrizio derivative operator is given as

$$
\begin{aligned}
U_{k+1}= & U_{0}+\left(\frac{1-\beta}{N(\beta)}+\frac{3 h}{2 N(\beta)}\right) L_{1}\left(t_{k}, \eta\left(t_{k}\right)\right) \\
& -\left(\frac{1-\beta}{N(\beta)}+\frac{\beta h}{2 N(\beta)}\right) L_{1}\left(t_{k-1}, \eta\left(t_{k-1}\right)\right),
\end{aligned}
$$

where

$$
\begin{aligned}
L_{1}(t, U)= & \varepsilon-d U-\alpha U V, \\
I_{k+1}=I_{0} & +\left(\frac{1-\beta}{N(\beta)}+\frac{3 h}{2 N(\beta)}\right) L_{2}\left(t_{k}, \eta\left(t_{k}\right)\right) \\
& -\left(\frac{1-\beta}{N(\beta)}+\frac{\beta h}{2 N(\beta)}\right) L_{2}\left(t_{k-1}, \eta\left(t_{k-1}\right)\right),
\end{aligned}
$$

where

$$
\begin{aligned}
L_{2}(t, I)= & \alpha U V-s I, \\
V_{k+1}= & V_{0}+\left(\frac{1-\beta}{N(\beta)}+\frac{3 h}{2 N(\beta)}\right) L_{3}\left(t_{k}, \eta\left(t_{k}\right)\right) \\
& -\left(\frac{1-\beta}{N(\beta)}+\frac{\beta h}{2 N(\beta)}\right) L_{3}\left(t_{k-1}, \eta\left(t_{k-1}\right)\right),
\end{aligned}
$$

where

$$
L_{3}(t, V)=c I-\delta V
$$

\section{Fractional HIV-1 Model in Atangana- Baleanu Sense}

The fractional mathematical model of HIV-1 infection in Atangana-Baleanu sense is given as follows.

$$
\begin{aligned}
& { }_{0}^{A B C} \zeta_{t}^{\beta} U(t)=\varepsilon-d U-\alpha U V, U(0)=\gamma_{1}, \\
& { }_{0}^{A B C} \zeta_{t}^{\beta} I(t)=\alpha U V-s I, I(0)=\gamma_{2}, \\
& { }_{0}^{A B C} \zeta_{t}^{\beta} V(t)=c I-\delta V, V(0)=\gamma_{3},
\end{aligned}
$$

here, ${ }_{0}^{A B C} \zeta_{t}^{\beta}$ is the Atangana-Baleanu fractional derivative of order $\beta$. 


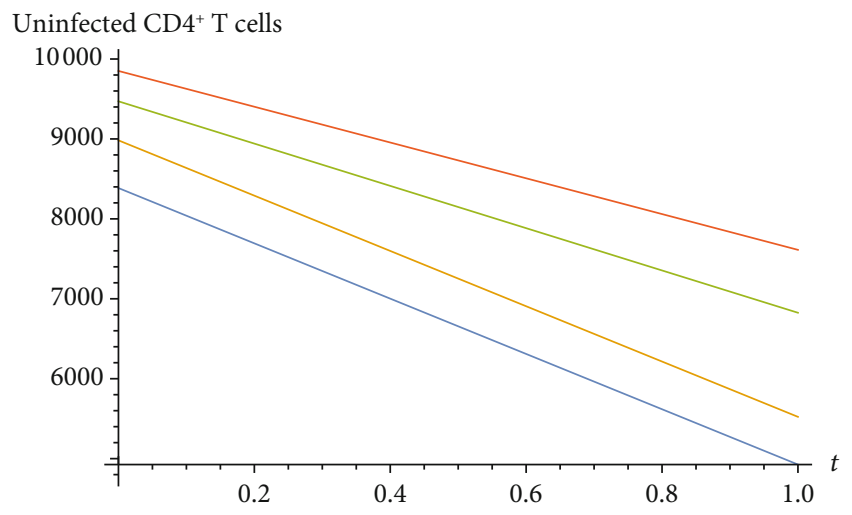

(a)

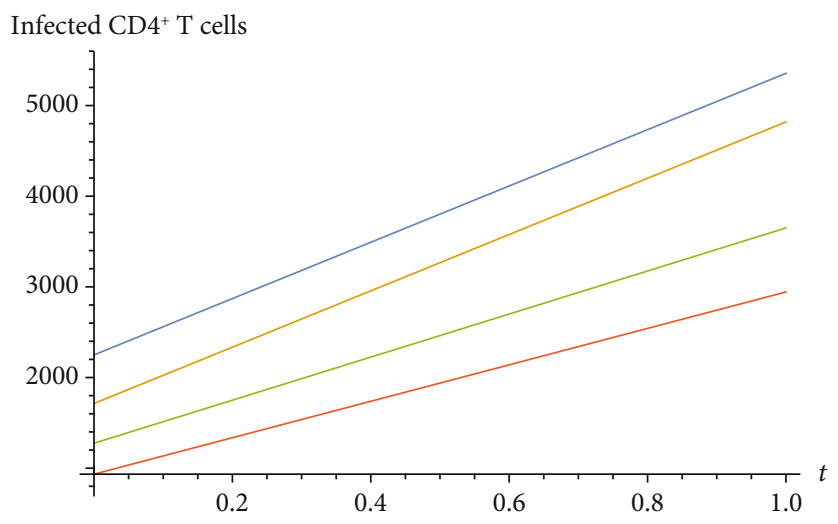

(b)

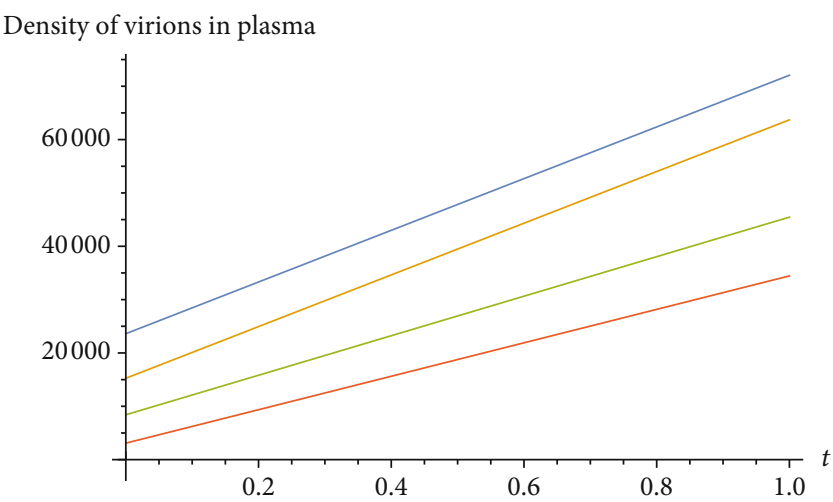

(c)

Figure 1: The graphical results to show the (a) uninfected CD4 ${ }^{+} \mathrm{T}$ cells, (b) infected CD4 ${ }^{+} \mathrm{T}$ cells, and (c) density of virions in plasma for different order fractional derivatives in sense of Caputo-Fabrizio operator, where blue represents graph for $\beta=0.3$, yellow for $\beta=0.5$, green for $\beta=0.7$, and red for $\beta=0.9$, respectively.

\subsection{Existence and Uniqueness of the Solution}

Theorem 6. The kernels

$$
\begin{gathered}
L_{1}(t, U)=\varepsilon-d U-\alpha U V, \\
L_{2}(t, I)=\alpha U V-s I, \\
L_{3}(t, V)=c I-\delta V,
\end{gathered}
$$

satisfy the Lipschitz condition and contractions if following hold:

$$
\begin{aligned}
& 0<k_{1}<1, \\
& 0<k_{2}<1, \\
& 0<k_{3}<1,
\end{aligned}
$$

where $k_{1}=d+n_{1}, k_{2}=s$, and $k_{3}=\delta$.

Proof. Consider the kernel

$$
L_{1}(t, U)=\varepsilon-d U-\alpha U V
$$

Let $U$ and $U_{1}$ be two functions, then

$$
\begin{aligned}
\left\|L_{1}(t, U)-L_{1}\left(t, U_{1}\right)\right\|= & \|(\varepsilon-d U-\alpha U V) \\
& -\left(\varepsilon-d U_{1}-\alpha U_{1} V\right) \| \\
\leq & d\left\|U-U_{1}\right\|+\alpha\|V\|\left\|U-U_{1}\right\| .
\end{aligned}
$$

Let $n_{1}=\max _{t}\|V(t)\|$, then

$$
\left\|L_{1}(t, U)-L_{1}\left(t, U_{1}\right)\right\| \leq k_{1}\left\|U(t)-U_{1}(t)\right\|
$$

where

$$
k_{1}=d+n_{1} \alpha
$$

Hence, this proves Lipschitz's condition for $L_{1}(t, U)$, and if $0<k_{1}<1$, then this proves contraction for $L_{1}(t, U)$. Similarly, we can prove the result for the kernels $L_{2}(t, I)$ and $L_{3}($ $t, V)$. 


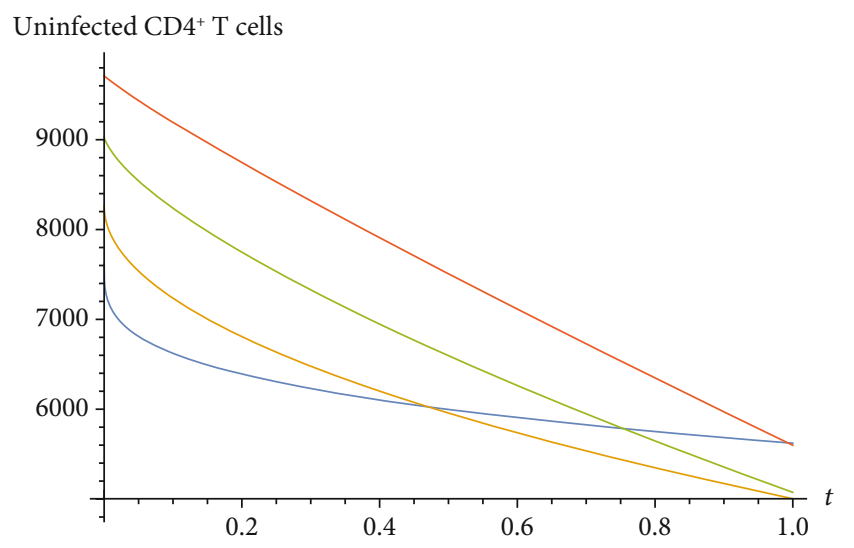

(a)

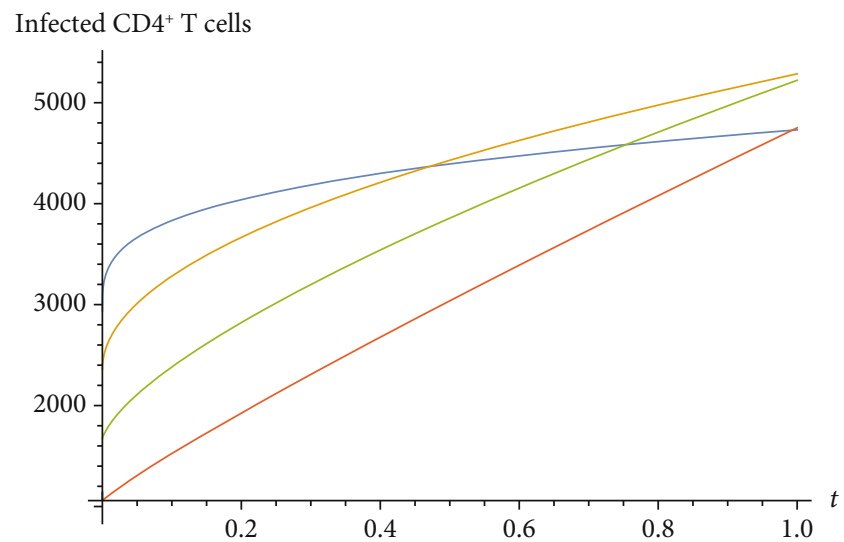

(b)

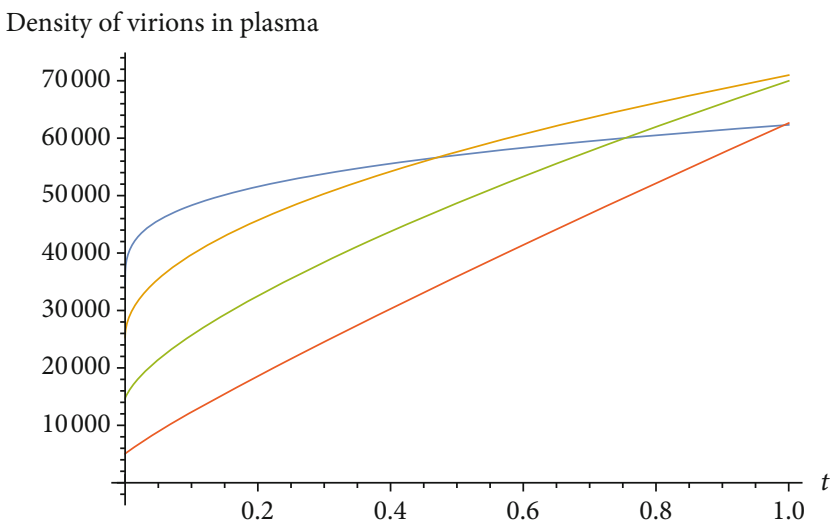

(c)

Figure 2: The graphical results to show the (a) uninfected $\mathrm{CD} 4^{+} \mathrm{T}$ cells, (b) infected $\mathrm{CD} 4^{+} \mathrm{T}$ cells, and (c) density of virions in plasma for different order fractional derivatives in sense of $\mathrm{ABC}$ operator, where blue represents graph for $\beta=0.3$, yellow for $\beta=0.5$, green for $\beta=$ 0.7 , and red for $\beta=0.9$, respectively.

Theorem 7. The time fractional HIV-1 infection model

$$
\begin{gathered}
{ }_{0}^{A B C} \zeta_{t}^{\beta} U(t)=\varepsilon-d U-\alpha U V, U(0)=\gamma_{1}, \\
{ }_{0}^{A B C} \zeta_{t}^{\beta} I(t)=\alpha U V-s I, I(0)=\gamma_{2}, \\
{ }_{0}^{A B C} \zeta_{t}^{\beta} V(t)=c I-\delta V, V(0)=\gamma_{3},
\end{gathered}
$$

possesses a unique solution under the conditions that we are able to search $t_{\max }$ which satisfies

$$
\frac{1-\beta}{N(\beta)} k_{i}+\frac{t_{\max }^{\beta}}{N(\beta) \Gamma(\beta)} k_{i}<1, \text { for } i=1,2,3,
$$

where $k_{1}=d+n_{1}, k_{2}=s$, and $k_{3}=\delta$.

Proof. We prove the result for equation (28).
Consider equation (28)

$$
{ }_{0}^{A B C} \zeta_{t}^{\beta} U(t)=\varepsilon-d U-\alpha U V, U(0)=\gamma_{1} .
$$

Let $L_{1}(t, U)=\varepsilon-d U-\alpha U V$.

Then, equation (32) can be written as

$$
{ }_{0}^{A B C} \zeta_{t}^{\beta} U(t)=L_{1}(t, U) .
$$

Using Theorem 5, we get

$$
\begin{aligned}
U(t)= & U_{0}+\frac{1-\beta}{N(\beta)} L_{1}(t, U(t)) \\
& +\frac{\beta}{N(\beta) \Gamma(\beta)} \int_{0}^{t}(t-\tau)^{\beta-1} L_{1}(\tau, U(\tau)) d \tau .
\end{aligned}
$$




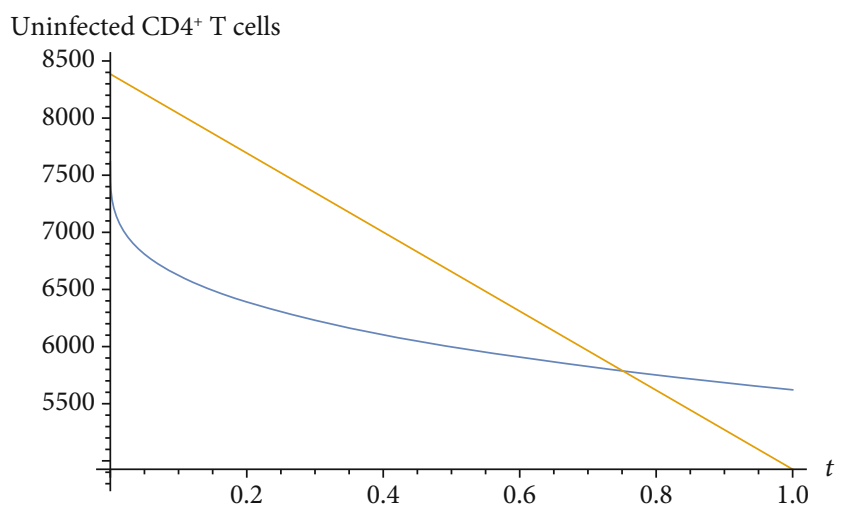

(a) $\beta=0.3$

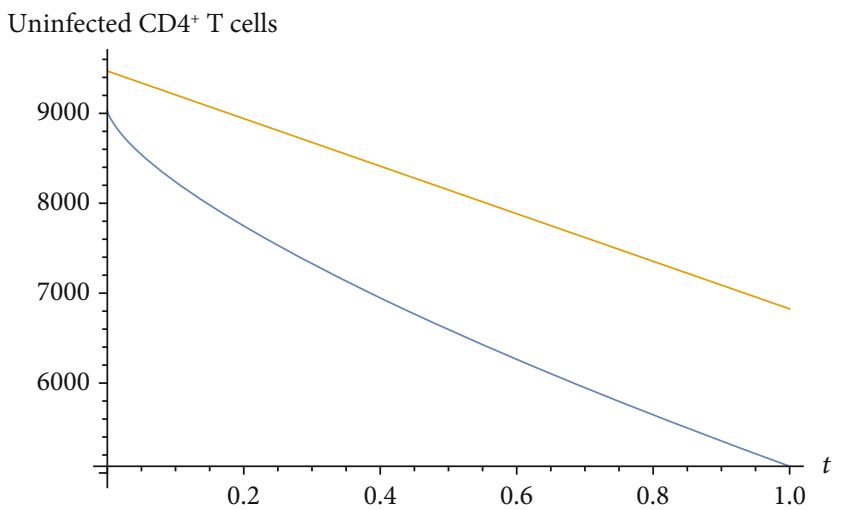

(c) $\beta=0.7$

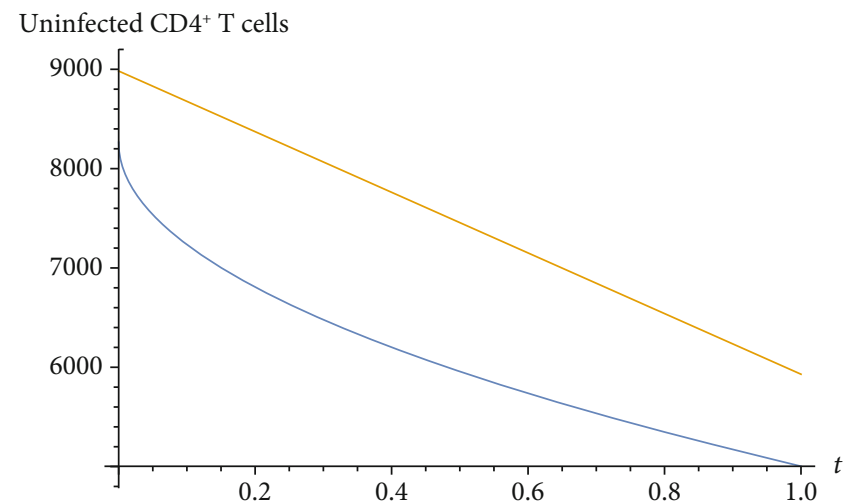

(b) $\beta=0.5$

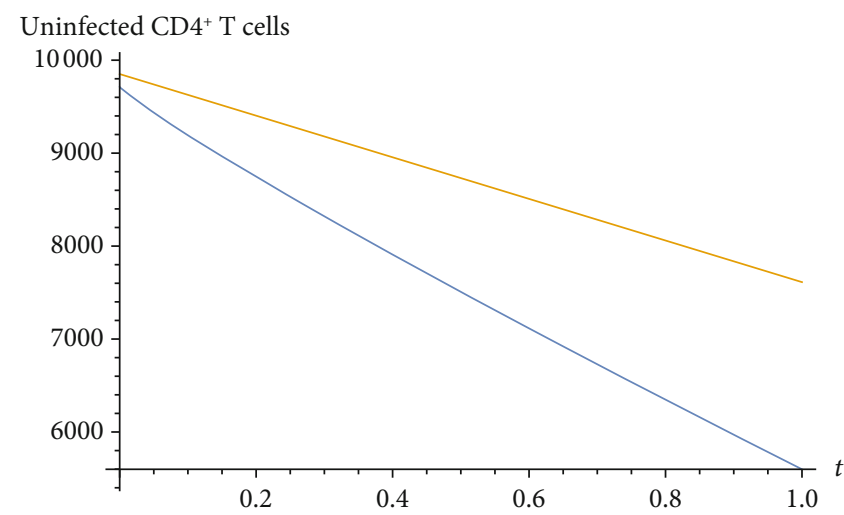

(d) $\beta=0.9$

Figure 3: The comparison of the behavior of uninfected CD4 ${ }^{+} \mathrm{T}$ cells in Caputo-Fabrizio and Atangana Baleanu sense for different order fractional derivatives, where blue colour represents graph for $\mathrm{ABC}$ operator and orange represents graph for $\mathrm{CF}$ operator.

Let $J=(0, T)$ and define an operator $Z: C\left(J, \mathbb{R}^{3}\right) \longrightarrow C$ $\left(J, \mathbb{R}^{3}\right)$ such that

$$
\begin{aligned}
Z[U(t)] & =U_{0}+\frac{1-\beta}{N(\beta)} L_{1}(t, U(t)) \\
& +\frac{\beta}{N(\beta) \Gamma(\beta)} \int_{0}^{t}(t-\tau)^{\beta-1} L_{1}(\tau, U(\tau)) d \tau .
\end{aligned}
$$

So equation (34) can be seen as $Z[U(t)]=U(t)$. Define the supremum norm on $J$ as $\|U\|=\operatorname{Sup}_{t \in J}|U(t)|$. Then, $C\left(J, \mathbb{R}^{3}\right)$ and $\|$.$\| define a Banach Space. Finally, consider$

$$
\begin{aligned}
& Z\left[U_{1}(t)\right]-Z\left[U_{2}(t)\right]=\frac{1-\beta}{N(\beta)}\left(L_{1}\left(t, U_{1}(t)\right)-L_{2}\left(t, U_{2}(t)\right)\right) \\
& \quad+\frac{\beta}{N(\beta) \Gamma(\beta)} \int_{0}^{t}(t-\tau)^{\beta-1}\left(L_{1}\left(\tau, U_{1}(\tau)\right)\right. \\
& \left.\quad-L_{1}\left(\tau, U_{1}(\tau)\right)\right) d \tau .
\end{aligned}
$$

Taking modulus on both sides of equation (36) and using triangle inequality, we get

$$
\begin{aligned}
& \left|Z\left[U_{1}(t)\right]-Z\left[U_{2}(t)\right]\right| \\
& \leq \frac{1-\beta}{N(\beta)}\left|\left(L_{1}\left(t, U_{1}(t)\right)-L_{2}\left(t, U_{2}(t)\right)\right)\right| \\
& \quad+\frac{\beta}{N(\beta) \Gamma(\beta)} \int_{0}^{t} \mid(t-\tau)^{\beta-1}\left(L_{1}\left(\tau, U_{1}(\tau)\right)\right. \\
& \left.\quad-L_{1}\left(\tau, U_{1}(\tau)\right)\right) d \tau \mid .
\end{aligned}
$$

Lastly, using the fact that the kernel $L_{1}(t, U(t))$ satisfies Lipschitz condition, we get

$$
\left|Z\left(U_{1}\right)-Z\left(U_{2}\right)\right| \leq\left(\frac{1-\beta}{N(\beta)} k_{1}+\frac{t_{\max }^{\beta}}{N(\beta) \Gamma(\beta)} k_{1}\right)\left|U_{1}-U_{2}\right| .
$$




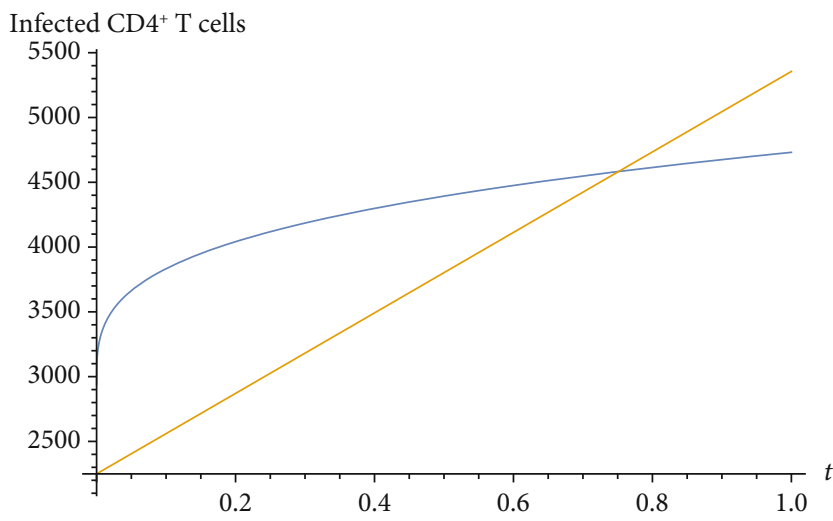

(a) $\beta=0.3$

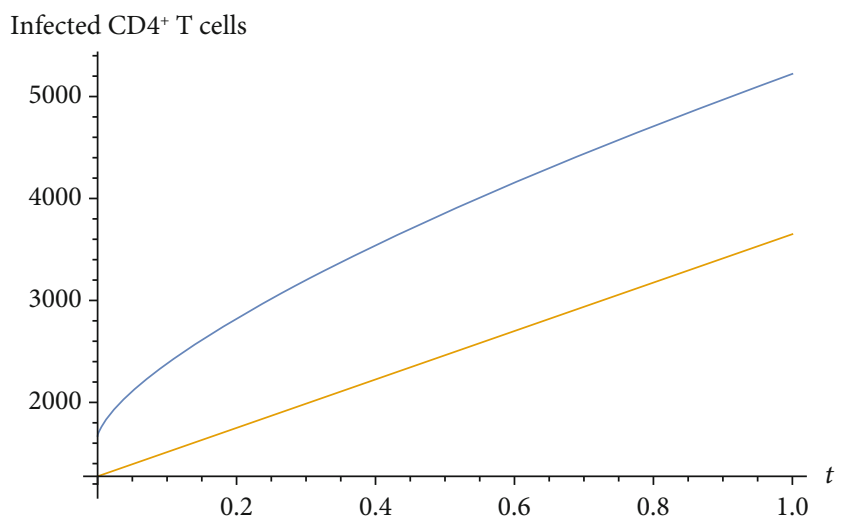

(c) $\beta=0.7$

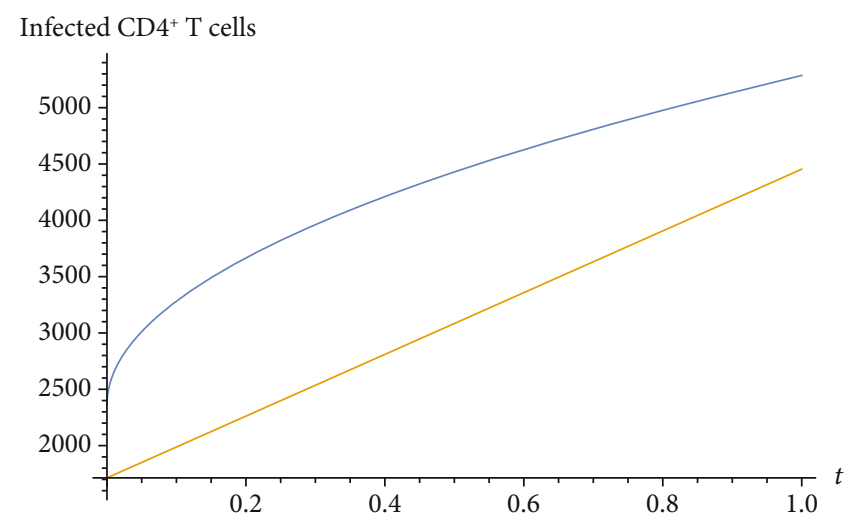

(b) $\beta=0.5$

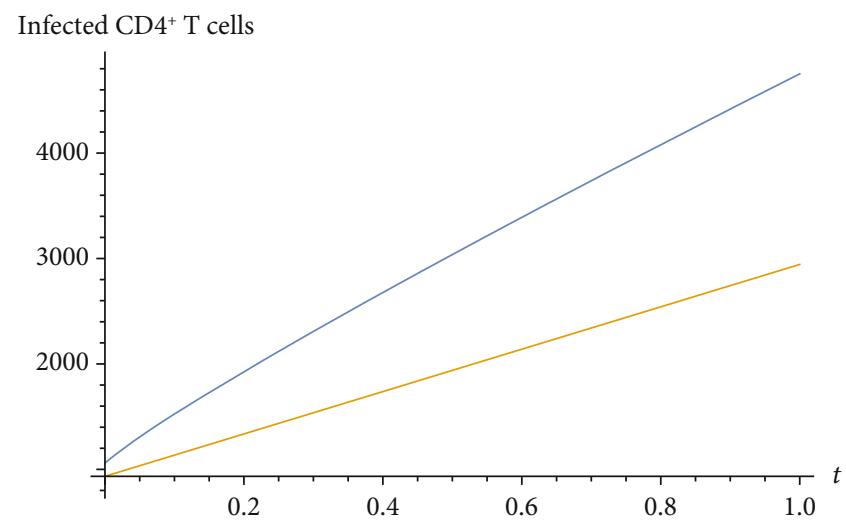

(d) $\beta=0.9$

Figure 4: The comparison of the behavior of infected $\mathrm{CD}^{+} \mathrm{T}$ cells in Caputo-Fabrizio and Atangana Baleanu sense for different order fractional derivatives, where blue colour represents graph for $\mathrm{ABC}$ operator and orange represents graph for $\mathrm{CF}$ operator.

Equation (38) is a contraction if

$$
\frac{1-\beta}{N(\beta)} k_{1}+\frac{t_{\max }^{\beta}}{N(\beta) \Gamma(\beta)} k_{1}<1
$$

Hence, using the Banach Fixed Point theorem, we govern the existence of a unique solution for the fractional model of HIV-1 infection in framework of Atangana-Baleanu derivative operator.

3.2. Derivation of Numerical Scheme in Sense of $A B C$ Derivative. Toufik and Atangana ([21, 22]) introduced a numerical scheme for solving fractional derivatives having nonsingular and nonlocal kernel. To illustrate the method, contemplate the following fractional differential equation:

$$
{ }_{0}^{A B C} \zeta_{t}^{\beta} \eta(t)=g(t, \eta(t)), t \geq 0, \eta(0)=\eta_{0} .
$$

Using Theorem 5, the above equation can be written as

$$
\begin{aligned}
\eta(t)-\eta(0)= & \frac{1-\beta}{N(\beta)} g(t, \eta(t))+\frac{\beta}{N(\beta) \Gamma(\beta)} \\
& \cdot \int_{0}^{t}(t-\tau)^{\beta-1} g(\tau, \eta(\tau)) d \tau .
\end{aligned}
$$

$$
\text { At point } t=t_{k+1} \text {, for } k=0,1,2 \cdots \text {, equation (41) becomes }
$$

$$
\begin{aligned}
\eta\left(t_{n+1}\right)-\eta(0)= & \frac{1-\beta}{N(\beta)} g\left(t_{k}, \eta\left(t_{k}\right)\right) \\
& +\frac{\beta}{N(\beta) \Gamma(\beta)} \int_{0}^{t_{k+1}}\left(t_{k+1}-\tau\right)^{\beta-1} g(\tau, \eta(\tau)) d \tau
\end{aligned}
$$

$$
\begin{aligned}
\eta_{k+1} & =\eta\left(t_{k+1}\right)=\eta(0)+\frac{1-\beta}{N(\beta)} g\left(t_{k}, \eta\left(t_{k}\right)\right) \\
& +\frac{\beta}{N(\beta) \Gamma(\beta)} \sum_{j=0}^{k} \int_{t_{j}}^{t_{j+1}}\left(t_{k+1}-\tau\right)^{\beta-1} g(\tau, \eta(\tau)) d \tau
\end{aligned}
$$

Considering $\mathrm{g}(\tau, \eta(\tau))$ through Lagrange polynomial interpolation,

$$
q_{n}=g(\tau, \eta(\tau))=\frac{\tau-t_{i-1}}{t_{i}-t_{i-1}} g\left(t_{i}, \eta_{t_{i}}\right)+\frac{\tau-t_{i}}{t_{i-1}-t_{i}} g\left(t_{i-1}, \eta_{t_{i-1}}\right) \text {. }
$$




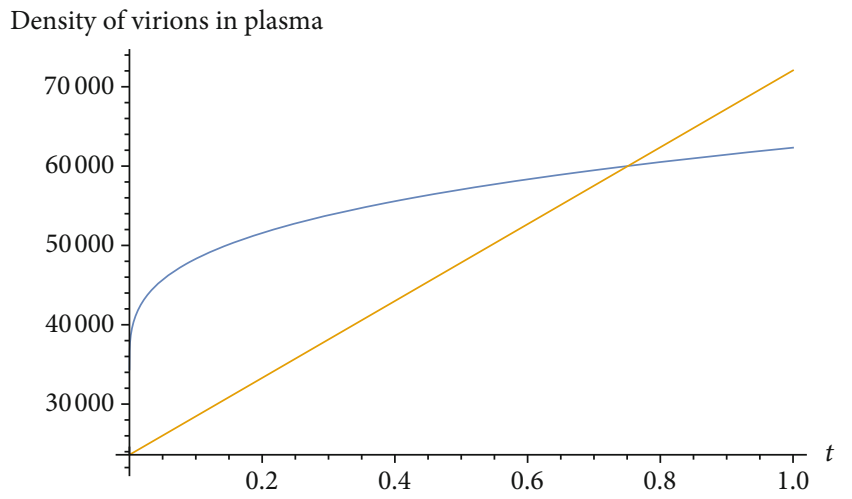

(a) $\beta=0.3$

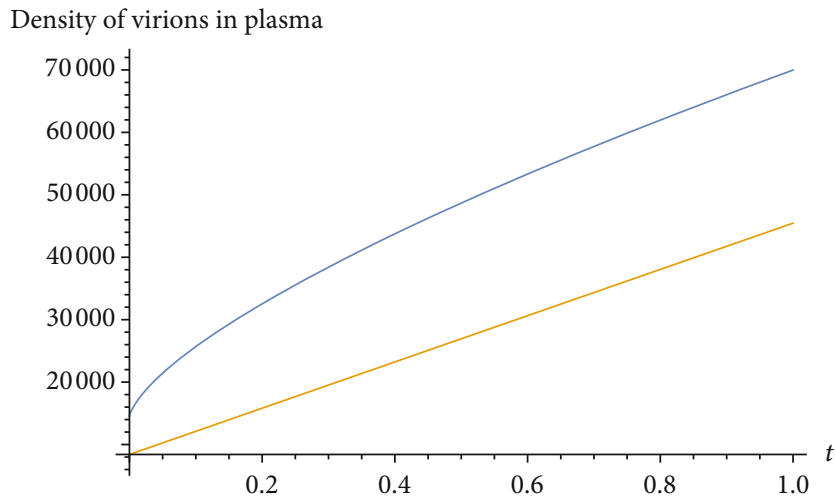

(c) $\beta=0.7$

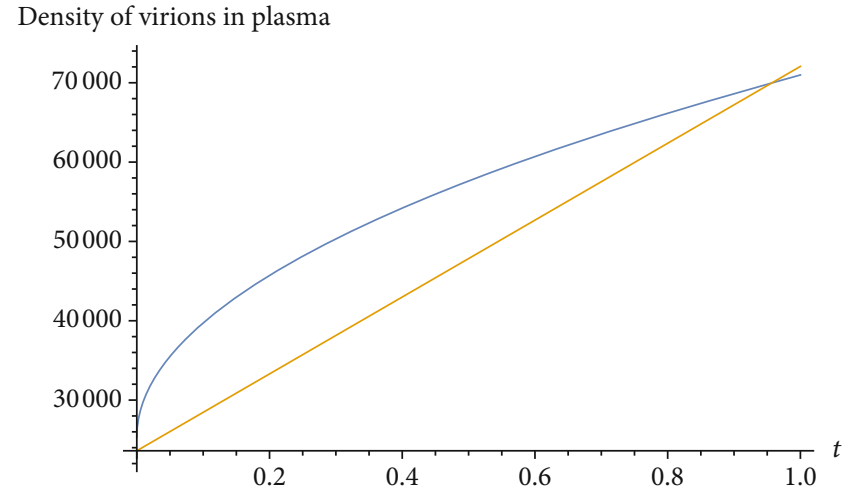

(b) $\beta=0.5$

Density of virions in plasma

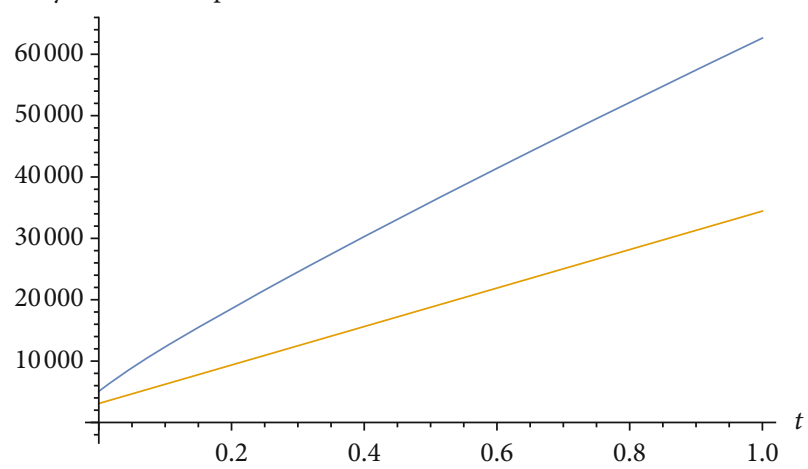

(d) $\beta=0.9$

FIgURE 5: The comparison of the behavior of density of virions in plasma in Caputo-Fabrizio and Atangana Baleanu sense for different order fractional derivatives, where blue colour represents graph for $\mathrm{ABC}$ operator and orange represents graph for $\mathrm{CF}$ operator.

Substituting the value of $g(\tau, \eta(\tau))$ in equation (43), we get

$$
\begin{aligned}
\eta_{k+1}= & \eta(0)+\frac{1-\beta}{N(\beta)} g\left(t_{k}, \eta\left(t_{k}\right)\right)+\frac{\beta}{N(\beta) \Gamma(\beta)} \sum_{i=0}^{k} \\
& \cdot\left(\frac{g\left(t_{i}, \eta\left(t_{i}\right)\right)}{h} \int_{t_{i}}^{t_{i+1}}\left(t-t_{i-1}\right)\left(t_{k+1}-t\right)^{\beta-1} d t\right. \\
& \left.-\frac{g\left(t_{i-1}, \eta\left(t_{i-1}\right)\right)}{h} \int_{t_{i}}^{t_{i+1}}\left(t-t_{i-1}\right)\left(t_{k+1}-t\right)^{\beta-1} d t\right) .
\end{aligned}
$$

Substituting $h=t_{i}-t_{i-1}$ and on solving, we get

$$
\begin{aligned}
\eta_{k+1}= & \eta_{0}+\frac{1-\beta}{N(\beta)} g\left(t_{k}, \eta\left(t_{k}\right)\right)+\frac{\beta}{N(\beta)} \sum_{i=0}^{k} \\
& \cdot\left[\frac { h ^ { \beta } g ( t _ { i } , \eta _ { t _ { i } } ) } { \Gamma ( \beta + 2 ) } \left((k+1-i)^{\beta}(k-i+2+\beta)\right.\right. \\
& \left.-(k-i)^{\beta}(k-i+2+2 \beta)\right)-\frac{h^{\beta} g\left(t_{i-1}, \eta\left(t_{i-1}\right)\right)}{\Gamma(\beta+2)} \\
& \left.\cdot\left((k+1-i)^{\beta+1}-(k-i)^{\beta}(k-i+1+\beta)\right)\right] .
\end{aligned}
$$

Using the aforementioned concept of numerical scheme, the numerical scheme for the fractional model of HIV-1 infection in framework of Atangana-Baleanu derivative operator is given as

$$
\begin{aligned}
U_{k+1}= & U_{0}+\frac{1-\beta}{N(\beta)} L_{1}\left(t_{k}, U\left(t_{k}\right)\right) \\
& +\frac{\beta}{N(\beta)} \sum_{i=0}^{k}\left[\frac{h^{\beta} L_{1}\left(t_{i}, U_{t_{i}}\right)}{\Gamma(\beta+2)}\right. \\
& \cdot\left((k+1-i)^{\beta}(k-i+2+\beta)\right. \\
& \left.-(k-i)^{\beta}(k-i+2+2 \beta)\right) \\
& -\frac{h^{\beta} L_{1}\left(t_{i-1}, U\left(t_{i-1}\right)\right)}{\Gamma(\beta+2)} \\
& \left.\cdot\left((k+1-i)^{\beta+1}-(k-i)^{\beta}(k-i+1+\beta)\right)\right],
\end{aligned}
$$

where

$$
L_{1}(t, U)=\varepsilon-d U-\alpha U V
$$




$$
\begin{aligned}
I_{k+1}= & I_{0}+\frac{1-\beta}{N(\beta)} L_{2}\left(t_{k}, I\left(t_{k}\right)\right)+\frac{\beta}{N(\beta)} \sum_{i=0}^{k} \\
& \cdot\left[\frac { h ^ { \beta } L _ { 2 } ( t _ { i } , I _ { t _ { i } } ) } { \Gamma ( \beta + 2 ) } \left((k+1-i)^{\beta}(k-i+2+\beta)\right.\right. \\
& \left.-(k-i)^{\beta}(k-i+2+2 \beta)\right)-\frac{h^{\beta} L_{2}\left(t_{i-1}, I\left(t_{i-1}\right)\right)}{\Gamma(\beta+2)} \\
& \left.\cdot\left((k+1-i)^{\beta+1}-(k-i)^{\beta}(k-i+1+\beta)\right)\right],
\end{aligned}
$$

where,

$$
\begin{gathered}
L_{2}(t, I)=\alpha U V-s I \\
V_{k+1}=V_{0}+\frac{1-\beta}{N(\beta)} L_{3}\left(t_{k}, V\left(t_{k}\right)\right)+\frac{\beta}{N(\beta)} \sum_{i=0}^{k} \\
\cdot\left[\frac { h ^ { \beta } L _ { 3 } ( t _ { i } , V _ { t _ { i } } ) } { \Gamma ( \beta + 2 ) } \left((k+1-i)^{\beta}(k-i+2+\beta)\right.\right. \\
\left.-(k-i)^{\beta}(k-i+2+2 \beta)\right)-\frac{h^{\beta} L_{3}\left(t_{i-1}, V\left(t_{i-1}\right)\right)}{\Gamma(\beta+2)} \\
\left.\cdot\left((k+1-i)^{\beta+1}-(k-i)^{\beta}(k-i+1+\beta)\right)\right],
\end{gathered}
$$

where,

$$
L_{3}(t, V)=c I-\delta V
$$

\section{Conclusion}

The graphical representation shows the change in the number of uninfected $\mathrm{CD}^{+}{ }^{+} \mathrm{T}$ cells, infected $\mathrm{CD} 4^{+} \mathrm{T}$ cells and the density of virions in plasma with respect to time. We have also inferred the effect of different fractional order derivatives to see the change in the number of uninfected $\mathrm{CD} 4^{+} \mathrm{T}$ cells, infected $\mathrm{CD}^{+}{ }^{+} \mathrm{T}$ cells and the density of virions in plasma with respect to time. From Figures 1 and 2, we see that on increasing the value of $\beta$ ( $\beta$ close to 1 ), the number of uninfected $\mathrm{CD}^{+} \mathrm{T}$ cells increases well while the number of infected $\mathrm{CD}^{+}{ }^{+} \mathrm{T}$ cells and the density of virions in plasma decreases. In Figures 3-5, we have shown the graphical comparison between the Caputo-Fabrizio operator and the Atanagana-Baleanu derivative operator. We have seen that on decreasing the value of $\beta$, the number of uninfected $\mathrm{CD}^{+} \mathrm{T}$ cells decreases well for the Atangana-Baleanu $(\mathrm{ABC})$ operator as compared to the Caputo-Fabrizio (CF) operator while the number of infected $\mathrm{CD}^{+}{ }^{+} \mathrm{T}$ cells and the density of virions in plasma increases well for the $\mathrm{ABC}$ operator than the $\mathrm{CF}$ operator. This shows that $\mathrm{ABC}$ derivative operator provides better results than the $\mathrm{CF}$ derivative operator.

\section{Data Availability}

No data were used to support this study.

\section{Conflicts of Interest}

There is no conflict of interest.

\section{Authors' Contributions}

All authors contributed in writing the draft and the software, and all reviewed and approved the final version of the manuscript.

\section{Acknowledgments}

The authors extend their appreciation to the Deanship of Scientific Research at King Saud University for funding this work through research group no (RG-1437-017).

\section{References}

[1] What is HIV?U.S. Department of Veterans Affairshttp://hiv.va .gov/patient/basics/what-is-HIV.asp.

[2] A. A. Okoye and L. J. Picker, "CD4+ T-cell depletion in HIV infection: mechanisms of immunological failure," Immunological Reviews, vol. 254, no. 1, pp. 54-64, 2013.

[3] S. Kumar, R. Kumar, J. Singh, K. S. Nisar, and D. Kumar, "An efficient numerical scheme for fractional model of HIV-1 infection of CD4+ T-cells with the effect of antiviral drug therapy," Alexandria Engineering Journal, vol. 59, no. 4, pp. 20532064, 2020.

[4] K. S. Miller and B. Ross, An Introduction to the Fractional Calculus and Fractional Differential Equations, A WileyInterscience Publication, New York, NY, USA, 1993.

[5] K. B. Oldham and J. Spanier, "The fractional calculus. Theory and applications of differentiation and integration to arbitrary order," in With an annotated chronological bibliography by Bertram Ross, p. xiii+234, Mathematics in Science and Engineering, Academic Press, New York-London, 1974.

[6] A. A. Kilbas, H. M. Srivastava, and J. J. Trujillo, Theory and Applications of Fractional Differential Equations, North-Holland Mathematics Studies, Elsevier, 2006.

[7] I. Podlubny, Fractional Differential Equations. An Introduction to Fractional Derivatives, Fractional Differential Equations, to Methods of Their Solution and Some of Their Applications, Mathematics in Science and Engineering, Academic Press, 1999.

[8] Y. Zhou, Basic Theory of Fractional Differential Equations, World Scientific Publishing, 2014.

[9] H. R. Marasi and H. Aydi, "Existence and uniqueness results for two-term nonlinear fractional differential equations via a fixed point technique," Journal of Mathematics, vol. 2021, Article ID 6670176, 7 pages, 2021.

[10] H. A. Hammad, H. Aydi, and N. Mlaiki, "Contributions of the fixed point technique to solve the 2D Volterra integral equations, Riemann-Liouville fractional integrals, and AtanganaBaleanu integral operators," Advances in Difference Equations, vol. 2021, no. 1, 2021.

[11] H. Aydi, M. Jleli, and B. Samet, "On positive solutions for a fractional Thermostat model with a convex-concave source 
term via $\psi$-Caputo fractional derivative," Mediterranean Journal of Mathematics, vol. 17, no. 1, 2020.

[12] H. A. Hammad, H. Aydi, and Y. U. Gaba, "Exciting fixed point results on a novel space with supportive applications," Journal of Functional Spaces, vol. 2021, article 6613774, pp. 1-12, 2021.

[13] A. Alshabanat, M. Jleli, S. Kumar, and B. Samet, "Generalization of Caputo-Fabrizio fractional derivative and applications to electrical circuits," Frontiers in Physics, vol. 8, 2020.

[14] S. Kumar, S. Ghosh, M. S. M. Lotayif, and B. Samet, "A model for describing the velocity of a particle in Brownian motion by Robotnov function based fractional operator," Alexandria Engineering Journal, vol. 59, no. 3, pp. 1435-1449, 2020.

[15] X. J. Yang, M. Abdel-Aty, and C. Cattani, “A new general fractional-order derivataive with Rabotnov fractionalexponential kernel applied to model the anomalous heat transfer," Thermal Science, vol. 23, no. 3, Part A, pp. 1677-1681, 2019.

[16] A. Atangana, "On the new fractional derivative and application to nonlinear Fisher's reaction-diffusion equation," Applied Mathematics and Computation, vol. 273, pp. 948956, 2016.

[17] M. Caputo and M. Fabrizio, "A new definition of fractional derivative without singular kernel," Progress in Fractional Differentiation and Applications, vol. 1, no. 2, pp. 73-85, 2015.

[18] A. Atangana and D. Baleanu, "New fractional derivatives with nonlocal and nonsingular kernel: theory and application to heat transfer model," Thermal Science, vol. 20, no. 2, pp. 763-769, 2016.

[19] J. J. Nieto and J. Losada, "Properties of a new fractional derivative without singular kernel," Progress in Fractional Differentiation and Applications, vol. 1, no. 2, pp. 87-92, 2015.

[20] K. M. Altaf and A. Atangana, "Dynamics of Ebola disease in the framework of different fractional derivatives," Entropy, vol. 21, no. 3, p. 303, 2019.

[21] R. Gnitchogna and A. Atangana, "New two step Laplace Adam-Bashforth method for integer a noninteger order partial differential equations," Numerical Methods for Partial Differential Equations, vol. 34, no. 5, pp. 1739-1758, 2018.

[22] M. Toufik and A. Atangana, "New numerical approximation of fractional derivative with non-local and non-singular kernel: application to chaotic models," The European Physical Journal Plus, vol. 132, no. 10, p. 444, 2017. 